\title{
The disturbance of hemostasis induced by hyperhomocysteinemia; the role of antioxidants
}

\author{
Joanna Malinowska, Joanna Kolodziejczyk and Beata Olas ${ }^{\bowtie}$ \\ Department of General Biochemistry, University of Łódź, Łódź, Poland
}

\begin{abstract}
Elevated concentration of homocysteine (Hcy) in human tissues, definied as hyperhomocysteinemia has been correlated with some diseases, such as cardiovascular, neurodegenerative, and kidney disorders. Homocysteine occurs in human blood plasma in several forms, including the most reactive one, the homocysteine thiolactone (HTL) - a cyclic thioester, which represents up to $0.29 \%$ of total plasma Hcy. In the article, the effects of hyperhomocysteinemia on the complex process of hemostasis, which regulates the flowing properties of blood, are described. Possible interactions of homocysteine and its different derivatives, including homocysteine thiolactone, with the major components of hemostasis such as endothelial cells, blood platelets, plasmatic fibrinogen and plasminogen, are also discussed. Modifications of hemostatic proteins ( $\mathrm{N}$-homocysteinylation or S-homocysteinylation) induced by Hcy or its thiolactone seem to be the main cause of homocysteine biotoxicity in hemostatic abnormalities. It is suggested that Hcy and HTL may also act as oxidants, but various polyphenolic antioxidants are able to inhibit the oxidative damage induced by Hcy or HTL. We also discuss the role of phenolic antioxidants in hyperhomocysteinemia -induced changes in hemostasis.
\end{abstract}

Key words: hyperhomocysteinemia, homocysteine, homocysteine thiolactone, hemostasis, blood platelets, fibrinogen, plasminogen, antioxidant

Received: 28 September, 2011; revised: 23 February, 2012; accepted: 04 April, 2012; available on-line: 11 May, 2012

\section{INTRODUCTION}

L-Homocysteine (Hcy) is an endogenous amino acid, containing a free thiol group, which in healthy cells is involved in methionine and cysteine synthesis/resynthesis. Indirectly, Hcy participates in methyl, folate, and cellular thiol metabolism (D'Angelo \& Selhub, 1997). Approximately $80 \%$ of total plasma Hcy is protein-bound, and only a small amount exists as a free reduced Hcy (about $0.1 \mu \mathrm{M})$. The majority of the unbound fraction of Hcy is oxidized, and forms dimers (homocystine) or mixed disulphides consisting of cysteine and Hcy (Table 1) (Mansoor et al., 1992; Ramakrishnan et al., 2006).

Over the years, several theories concerning the toxicity of Hcy have been elaborated, but despite the efforts, none fully explains the toxicity of this compound. However, two main pathways of Hcy biotoxicity are discussed: 1) Hcy-dependent oxidative stress and 2) Hcy-induced protein structure modifications, named homocysteinylation. In the first case, oxidative stress is generated during oxidation of the free thiol group of Hcy, when
Hcy binds via a disulphide bridge with plasma proteins - mainly albumin, or with other low-molecular plasma thiols or with a second Hcy molecule. Oxidation of Hcy may induce the subsequent oxidation of proteins, lipids and nucleic acids (Zou \& Banerjee, 2005). Accumulation of oxidized biomolecules alters the biological functions of many cellular pathways. In the second case, two main types of homocysteinylation exist: S-homocysteinylation and N-homocysteinylation; both of them can be considered as posttranslational protein modifications. S-homocysteinylation occurs when Hcy reacts, by its free thiol group, with another free thiol derived from a cysteine residue in a protein molecule. These changes can alter the thiol-dependent redox status of proteins (Sengupta et al., 2001; Jakubowski 2004). N-homocysteinylation takes place after acylation of the free $\varepsilon$-amino lysine groups of proteins by the most reactive form of Hcy - its cyclic thioester (Hcy thiolactone - HTL), representing up to $0.29 \%$ of total plasma Hcy (Jakubowski, 1999 and 2003; Perla-Kajan et al., 2007). The chemical structure of Hcy and its thiolactone is presented in Fig. 1. In human blood, N-homocysteinylated (N-Hcy-protein) and S-homocysteinylated proteins (S-Hcy-protein) such as $\mathrm{N}$ Hcy-hemoglobin, N-(Hcy-S-S-Cys)-albumin, and S-Hcyalbumin were described (Jakubowski, 2002; 2005; 2006; Chwatko \& Jakubowski, 2005a; 2005b; Perla-Kajan et al., 2007). Moreover, also other pathways of Hcy biotoxicity may exist, i.e. apoptosis and excitotoxicity mediated through glutamate receptors.

Kang et al. (1992) classified several types of hyperhomocysteinemia, in relation to the total plasma Hcy concentration. They defined hyperhomocysteinemia as severe, for concentrations higher than $100 \mu \mathrm{M}$, intermediate for concentrations between 31 and $100 \mu \mathrm{M}$, and moderate for concentrations of $16-30 \mu \mathrm{M}$, and a reference total plasma Hcy range as 5 to $15 \mu \mathrm{M}$ (mean, 10 $\mu \mathrm{M})$. Lentz \& Haynes (2004) defined hyperhomocysteinemia as severe $(>100 \mu \mathrm{M})$, moderate $(30-100 \mu \mathrm{M})$ and mild $(10-30 \mu \mathrm{M})$. They defined the physiological range

e-mail: olasb@biol.uni.lodz.pl

Abbreviations: AARS, aminoacyl-tRNA synthetase; ADP, adenosine diphosphate; $C B S$, cystathionine $\beta$-synthase; CSE, cystathionine $\gamma$-lyase; Cys, cysteine; FN, fibronectin; GFR, glomerular filtration rate; Hcy, homocysteine; HTL, homocysteine thiolactone; HTLase, homocysteine thiolactonase; Lp(a), lipoprotein (a); Met, methionin; Met, methionine; MetRS- methionyl-t-RNA synthetase; MS, methionine synthase; MTHFR, methylenetetrahydrofolate reductase; $N O$, nitric oxide; $\mathrm{O}_{2}^{--}$, superoxide anion; PAF, platelet aggregating factor; PAl-1, plasminogen activator inhibitor type-1; PAl-2, plasminogen activator inhibitor type-2; PDI, protein disulphide isomerase; PON, paraoxonase 1; RNS, reactive nitrogen species; ROS, reactive oxygen species; SAH, S-adenosylhomocysteine; SAM, S-adenosylmethionine; SAM synthetase, methionine adenosyltransferase; t-PA, tissue plasminogen activator; TXA2, thromboxane A2; u-PA, urokinase plasminogen activator. 
Table 1. Different forms of homocysteine present in human blood.

*Total level of homocysteine - the term "total homocysteine" describes the pool of homocysteine released by reduction of all disulphide bonds in the sample (Perla-Kajan et al., 2007; Zimny, 2008; Manolescu et al., 2010, modified).

\begin{tabular}{ll}
\hline The form of Hcy & The concentration in human blood \\
\hline Homocysteine thiolactone (HTL) & $0-35 \mathrm{nM}$ \\
Protein N-linked homocysteine: N-Hcy-hemoglobin, N-(Hcy-S-S-Cys)-albumin & about $15.5 \mu \mathrm{M}: 12.7 \mu \mathrm{M}, 2.8 \mu \mathrm{M}$ \\
Protein S-linked homocysteine - S-Hcy-albumin & about $7.3 \mu \mathrm{M}^{*}$ \\
Homocystine (Hcy-S-S-Hcy) and combined with cysteine to from mixed disulphides (Hcy-S-S-Cys) & about $2 \mu \mathrm{M}^{*}$ \\
Free reduced Hcy & about $0.1 \mathrm{M}^{*}$ \\
\hline
\end{tabular}

of total plasma Hcy as the 2.5th to 97.5 th percentile interval for the analyzed group that corresponds to a lower limit of $5 \mu \mathrm{M}$, while the upper limit varies considerably between laboratories and different populations. As in the case of other biochemical parameters, a reference interval for Hcy which will take into account the influence of different non-modifiable and modifiable factors, should be established. The relationship between homocysteine and risk appears to hold for total plasma concentrations of homocysteine between 10 and $30 \mu \mathrm{M}$.

The elevated plasma level of Hcy may be a result of the genetic defects of enzymes involved in its metabolism, such as cystathionine- $\beta$-synthase (CBS), methionine synthase (MS) and methylenetetrahydrofolate reductase (MTHFR) (Brattstörm et al., 1998). As early as in the 1960 s it was noted that the risk of atherosclerosis is markedly increased in patients with homocystinuria, an inherited disease resulting from homozygous CBS deficiency and characterized by episodes of thromboembolism, mental retardation, lens dislocation, hepatic steatosis, and osteoporosis. Homocystinuria is associated with abnormal, very high concentrations of plasma homocysteine and methionine. Patients with homocystinuria have very severe hyperhomocysteinemia, with plasma homocysteine concentration reaching even $400 \mu \mathrm{M}$, and represent a very small proportion of the population (approximately 1 in 200,000 individuals). In patients with residual CBS activity (between $0 \%$ and $2 \%$ of normal), who represent approximately half of the cases of the homozygous homocystinuria, pyridoxine treatment can decrease hyperhomocysteinemia. Heterozygous lack of CBS, CBS mutations and polymorphism of the methylenetetrahydrofolate reductase gene are considered to be the most probable causes of hyperhomocysteinemia (Franken et al., 1994). In CBS, Ile278Thr and Gly307Ser are the most common mutations. If they occur homozygously, these mutations may lead to a significant elevation of plasma homocysteine level and to an increased risk of atherosclerosis of carotid arteries (Klerk et al., 2002). Thirty-three point mutations of the CBS gene,
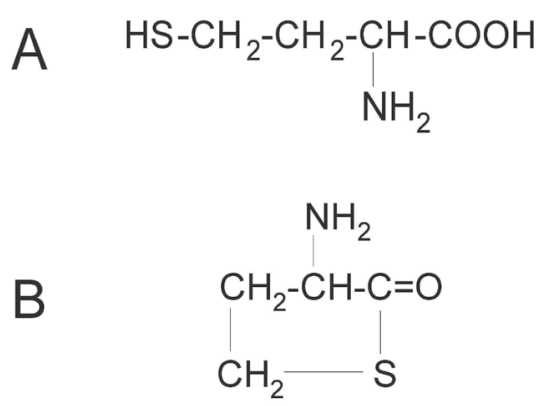

Figure 1. The chemical structure of homocysteine (A) and its thiolactone (B). which is located on chromosome 21 , have been identified. Among these mutations, the G919A transition, frequently encountered in the Irish population, resulting in the substitution of guanine by adenine in position 919, is observed in subjects who are refractory to vitamin $\mathrm{B}_{6}$ treatment. The T833C mutation, frequent in the German population, is observed in vitamin $B_{6}$ responders. People who are heterozygous for CBS deficiency have normal plasma homocysteine levels in $30 \%$ to $50 \%$ of cases (Lentz et al, 2000; Durand et al, 2001).

Methylenetetrahydrofolate reductase gene polymorphism involves substitution of cytosine by thymine in position $677(\mathrm{C} 677 \rightarrow \mathrm{T})$ and leads to the synthesis of a thermolabile enzyme form with decreased activity. Prevalence of this mutation is race dependent. In Asian and Caucasian populations (including Poland) there are $50 \%$ C7'T heterozygotes and 10-13\% T/T homozygotes, while in the Afro-American population this polymorphism is rare (Brattstörm et al., 1998; Bełtowski 2005). $\mathrm{T} / \mathrm{T}$ homozygotes have, on average, Hcy levels higher by $2.5 \mu \mathrm{M}$ than $\mathrm{C} / \mathrm{C}$ homozygotes. However, this difference has been observed in the case of folic acid and riboflavin deficiency in the diet (Bednarek-Tupikowska et al., 2004). It has also been observed that $A \rightarrow C$ transversion in position 1298 of the MTHFR gene results in the substitution of glutamate with alanine in the enzyme, which leads to its decreased activity (Chango et al., 2000). Nine thermostable mutations of the MTHFR gene, located on chromosome 1 , inherited as an autosomal recessive trait, could be responsible for its reduced activity. The two main mutations are the C599T and the G482A transitions. Hyperhomocysteinemia is only observed in the homozygous state. No vitamin treatment has been effective in these patients, although their hyperhomocysteinemia is less severe than in CBS deficient homozygotes.

Acquired hyperhomocysteinemia may result from various factors, such as lifestyle, diet, various diseases, medications, drugs, sex and age. Unbalanced diet, leading to significant deficiency of vitamins $\mathrm{B}_{2}, \mathrm{~B}_{6}, \mathrm{~B}_{12}$ and folic acid may cause an increase in plasma Hcy concentration. Additionally, alcohol, tobacco smoking and coffee also lead to increased plasma Hcy level. The difference observed in homocysteine level between men and women could be explained by body size or estrogen, the dependence of homocysteine concentration on age could result from the decline of the glomerular filtration rate (GFR) with increasing age. Also alimentary tract disorders connected with impaired absorption of vitamins $B_{2}, B_{6}$, $\mathrm{B}_{12}$ and folic acid may play an important role. In some countries, e.g. in the U.S.A., supplementation of food with folic acid is a common occurrence. In Poland, however, the food is not supplemented with folic acid and therefore the levels of total plasma homocysteine should 


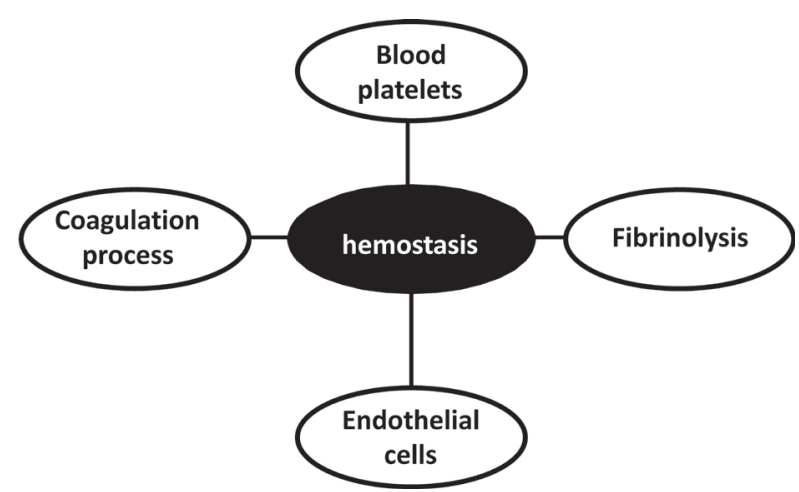

Figure 2. Different elements of hemostasis.

more closely depend on the dietary and genetic variation (Suwala et al., 2009).

Elevated plasma Hcy $(>15 \mu \mathrm{M}$; Hcy) is associated with an increased risk of cardiovascular diseases (Harpel et al., 1992; 1996; Boysen et al., 2003; Li et al., 2003; Karolczak \& Olas, 2009; Bloom et al., 2011; Cacciapuoti, 2011), such as thrombosis (Harpel et al., 1992; 1996; Matteo et al., 2010; Kamat et al., 2010) and thrombosisrelated diseases, such as stroke (Boysen et al., 2003; Li et al., 2003; Suwala et al., 2009). Every increase of 2.5 $\mu \mathrm{M}$ in plasma Hcy may be associated with an increase of stroke risk of about $20 \%$ (Clarke et al., 2002). Suwala et al. (2009) analyzed the occurrence of ischemic brain stroke in the population of Northern Poland with regard to risk factors of the disease. With the use of a modified HPLC method (described by Suwala et al., 2008), those authors demonstrated that hyperhomocysteinemia might be a risk factor of ischemic brain stroke, independent of other, conventional risk factors of this disease. Moreover, total plasma Hcy level above $20 \mu \mathrm{M}$ are associated with a nine-fold increase of the myocardial infarction and stroke risk, in comparison to the concentrations below $9 \mu \mathrm{M}$ (Nygard et al., 1997). The increase of Hcy concentration has been also found in other human pathologies, including neurodegenerative diseases (Ravaglia et al., 2005). However there are many different opinions on the biotoxicity of Hcy on the hemostasis process.

This review describes the effect of hyperhomocysteinemia on different components of hemostasis (blood platelets, endothelial cells, plasmatic fibrinogen and plasminogen (Fig. 2)) and hemostatic abnormalities (Fig. 3). The normal hemostatic mechanisms prevent the hemor-

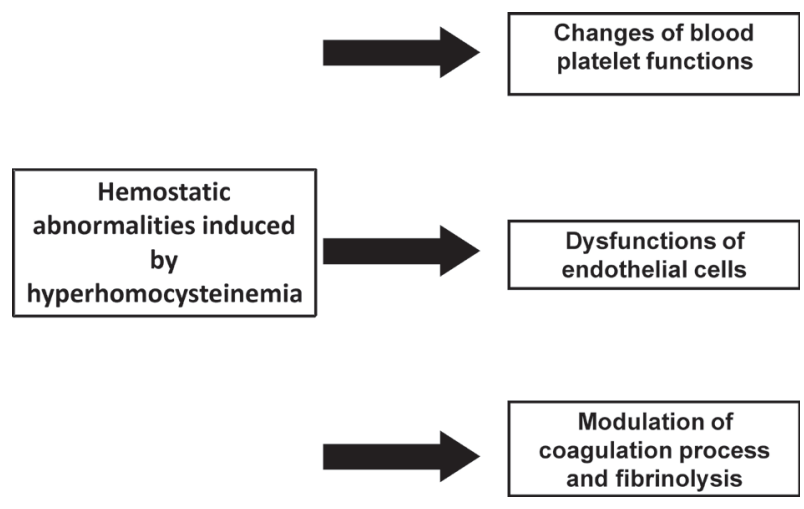

Figure 3. Hemostatic abnormalities induced by hyperhomocyste inemia. rhage and thrombosis by complex interactions between the blood vessel wall, blood platelets, coagulation factors and fibrinolytic proteins. The vascular endothelium maintains the balance between prevention and stimulation of platelet activation, coagulation and fibrinolysis and between vasoconstriction and vasodilation. In the present article, we also discuss the results of various experiments, performed to investigate the beneficial effects of phenolic antioxidants on changes in hemostasis induced by hyperhomocysteinemia.

\section{PRODUCTION AND METABOLISM OF HOMOCYSTEINE AND ITS THIOLACTONE}

Hcy is a homologue of the amino acid cysteine (Cys), differing by an additional methyl group. Hcy is formed as a result of the transformation of methionine (Met) to Cys in all types of animal cells, including human. In the reaction catalyzed by methionine adenosyltransferase (SAM synthetase) adenosine is transferred from ATP to Met to form S-adenosylmethionine (SAM). SAM is the major donor of methyl groups for various methylation reactions, therefore, is called "active methyl" or "active methionine". When a methyl group $\left(-\mathrm{CH}_{3}\right)$ is transferred by SAM synthetase to individual acceptors, SAM is converted to S-adenosylhomocysteine $(\mathrm{SAH})$. Then, $\mathrm{SAH}$ is hydrolyzed by a specific SAH hydrolase to adenosine and Hcy. Hcy is transported outside the cell and its concentration can be determined in plasma.

In human cells Hcy can be metabolised via two B-vitamins-dependent pathways: remethylation and transsulfuration. In the first pathway, Hcy may be remethylated to Met by MS, which uses 5-methyltetrahydrofolate and vitamin $\mathrm{B}_{12}$ as cofactors. The second pathway - transsulfuration - leads to cysteine. This pathway of Hcy metabolism is catalyzed by CBS and CSE, with pyridoxal-5 phosphate as a cofactor (active form of vitamin $\mathrm{B}_{6}$ ) (Fig. 4) (Perla-Kajan et al., 2007).

The synthesis of homocysteine thiolactone is associated with the activation of the amino acid by aminoacyl-tRNA synthetase (AARS). Hcy may also undergo erroneous activation, e.g. by methionyl-t-RNA synthetase (MetRS) (Jakubowski, 1990). In the first step of conversion of-Hcy to HTL, MetRS misactivates Hcy giving rise to homocysteinyl-adenylate. In the next phase, the homocysteine side chain thiol group reacts with the activated carboxyl group and HTL is produced. The level of HTL synthesis in cultured cells depends on Hcy and Met levels. HTL may be hydrolyzed nonenzymatically,

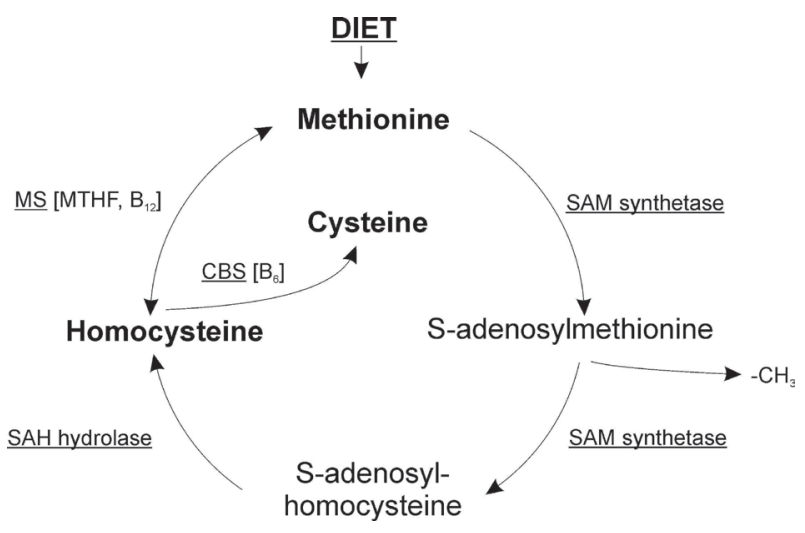

Figure 4. Metabolism of homocysteine.

Abbreviations: CBS, cystathionine $\beta$-synthase; CSE, cystathionine Y-lyase; MS, methionine synthase; 5-MTHF, 5-methyltetrahydrofolate. 
but it is mostly metabolized by plasma HTL hydrolase, also known as homocysteine thiolactonase (HTLase) or paraoxonase (PON 1) (Jakubowski, 2000; Perla-Kaján \& Jakubowski, 2010; Jakubowski \& Głowacki, 2011).

\section{HYPERHOMOCYSTEINEMIA AND CHANGES IN FIBRINOLYSIS AND COAGULATION PROCESS}

The fibrinolytic system comprises a proenzyme, plasminogen, which can be converted to the active enzyme, plasmin, by plasminogen activators. The main plasminogen activator in the intravascular fibrinolysis is tissue plasminogen activator (t-PA); another plasminogen activator - urokinase plasminogen activator (u-PA) is mostly involved in the extracellular proteolysis (Lijnen, 2002). Both u-PA and t-PA are serine proteinases that act directly on the peptide bond Arg560-Val561 of the plasminogen molecule, but the plasminogen activation process induced by t-PA occurs on the fibrin clot surface. The fibrinolytic activity of blood is regulated by specific inhibitors; the inhibition of fibrinolysis takes place at the level of plasminogen activation (by PA-inhibitors: plasminogen activator inhibitor type-1, -2; PAI1 or PAI-2) or at the level of plasmin activity (mainly by $\alpha_{2}$-antiplasmin) (Booth, 2000).

Hyperhomocysteinemia disturbs hemostasis and shifts the hemostatic mechanisms in favor of thrombosis. The recent reports indicate that the prothrombotic state observed in hyperhomocysteinemia may arise not only due to endothelium dysfunction or blood platelet and coagulation activation, but also due to impaired fibrinolysis (Kołodziejczyk et al., 2010). It has been shown, that Hcy-modified fibrinogen is more resistant to the fibrinolytic action (Undas et al., 2006). The studies of Freyburger et al. (1997) demonstrated that the increased level of total Hcy, induced by oral methionine load, may diminish the fibrinolytic activity of the euglobulin plasma fraction. Also the enzymes of the fibrinolytic system may be a potential target for homocysteine action (Colucci et al., 2008). Moreover, other clinical studies carried by Speidl et al. (2007) on cardiovascular disease patients after their first myocardial infarction, who also had hyperhomocysteinemia showed a reduced t-PA activity independently from cardiovascular risk factors and medical treatment. Authors suggest that homocysteine-lowering therapies may increase fibrinolytic activity and prevent atherothrombotic events in patients with cardiovascular diseases after the first myocardial infarction.

The other possible mechanism of the prothrombotic effect of homocysteine may be the direct blockade of the t-PA binding domain of annexin II, its major endothelial cell receptor (Hajjar et al., 1998). t-PA attaches to endothelial cells via the calcium-regulated phospholipidbinding protein annexin II and this interaction is inhibited by Hcy complexing with Cys9 within annexin II putative hexapeptide binding domain (LCKLSL). It has been estimated that Hcy at the concentration of about $11 \mu \mathrm{M}$ is able to inhibit t-PA binding to annexin II by as much as $50 \%$; this extent of the loss of fibrinolytic potential seems to be an important factor in the etiology of vascular disorders.

In further studies, Dassah and coworkers (2009) incubated annexin II in a purified protein system with homocysteine. Annexin II was modified by Hcy. This treatment blocked the ability of t-PA to bind to annexin II. Finally, incubation of cultured ECs with 35S-Hcy led to metabolic labeling of annexin II that was sensitive to protein reduction, suggesting a di- sulfide-mediated association between Cys8 and Hcy. These data revealed a binding domain for t-PA in the $\mathrm{N}$-terminal tail of annexin II, and showed its susceptibility to modification by Hcy, an agent highly associated with atherothrombotic vascular disease.

The impairment of fibrinolytic efficacy may be also a result of the enhanced level of lipoprotein (a) $(\mathrm{Lp}(\mathrm{a}))$, which is one of the common risk factors of atherosclerosis. Since $\operatorname{Lp}(\mathrm{a})$ contains apoprotein (a), with a sequence highly homologous to plasminogen, it can act as a competitive inhibitor of plasminogen. $\mathrm{Lp}$ (a) may bind to cellular plasminogen receptors and to fibrin(ogen) and competes for the binding of plasminogen to these molecules (Xue et al., 1999). According to the study of Harpel et al. (1992) Hcy promotes the attachment of lipoprotein (a) to fibrin, decreasing the possibility of plasminogen binding and activation. Nardulli et al. (2005) suggest the potential mechanism for the homocysteine-enhanced antifibrinolytic action of lipoprotein(a) in human plasma. According to these scientists, Hcy reduces the apo(a)/ apoB disulphide bond and causes the appearance of free apo(a) with high affinity for fibrin, which is able to strongly inhibit plasminogen binding. Furthermore, it seems very likely, that one of the prothrombotic mechanisms of homocysteine action is the modification of the fibrinogen structure (Acevedo et al., 2002). Fibrinogen is a key protein in blood coagulation. The most significant biological role of fibrinogen is related to its ability to form the scaffold of a blood clot and thereby prevent the loss of blood after injury. It is of greatest consequence for the maintenance of health that the capacity of the soluble fibrinogen to be converted into insoluble fibrin is meticulously regulated, and that stable fibrin is formed only when and where it is needed. Fibrinogen plays also a role in the physiological and pathological processes related to wound healing, tumor growth, and metastasis as well as defense mechanisms. In order to fulfill its numerous functions, fibrinogen interacts in highly specific ways with a large number of other proteins as well as low molecular weight cellular components, primarily in the blood stream or blood vessel walls. Human fibrinogen is subject to modification at a number of different sites both during and after biosynthesis. These modifications include oxidation, nitration, non-enzymatic glycation and homocysteinylation. Other studies have shown, that Hcy- and HTL-modified fibrinogen is more resistant to the fibrinolytic action (Undas et al., 2006; Malinowska et al., 2011). Homocysteine thiolactone-mediated changes in the fibrinogen molecule may increase the resistance of the fibrin clot to degradation. Studies by Sauls et al. (Sauls et al., 2003 and 2006) with the use of mass spectrometric analysis of Hcy fibrinogen showed homocysteinylation of twelve lysine residues. Moreover, several of them were localized close to t-PA and plasminogen binding sites. The authors suggested that the Hcy-induced modification of lysine might occur in vivo. Lysine residues in the fibrinogen molecule are crucial for the interaction with fibrinolytic enzymes, thus modification of these amino acids may lead to increased resistance of clots to lysis and contribute to the thrombotic tendency occurring in hyperhomocysteinemia. HTL can react with primary amines such as those in lysine, asparagine, arginine, and glutamine. Homocysteinylation introduces a new free sulfhydryl group into the protein, as well as alters the size of the modified amino acid. Under physiological conditions the reaction with the epsilon 
amino group of lysine is favored (Jakubowski, 1999; Hop \& Bakhitiar, 2002; Głowacki \& Jakubowski, 2004; Sikora et al., 2010; Marczak et al., 2011). Limited homocysteinylation induces the features of the homocysteinemia-associated dysfibrinogenemia including the formation of thin, tightly packed fibrin fibers and increased resistance to fibrinolysis. Fibrin formed from $\mathrm{N}$ - homocysteinylated fibrinogen also has a decreased ability to support activation of plasminogen by t-PA (Sauls et al., 2006).

Another hemostatic protein, susceptible for homocysteinylation is factor V (Undas et al., 1996). Homocysteine rapidly incorporates into its structure in vitro due the modification of cysteine(s) and formation of heterologous disulphide bonds. The homocysteine treatment of factor $\mathrm{V}$ protects $\alpha$-thrombin-derived factor $\mathrm{Va}$ from inactivation by supporting fibrinolysis.

\section{THE EFFECT OF HYPERHOMOCYSTEINEMIA ON BLOOD PLATELET FUNCTIONS}

Blood platelets are multiresponding cells, both with respect to the number of agonists and number of responses. They can be activated by different compounds including coagulation factors (thrombin), hormones (epinephrine, vasopressin, low-molecular-weight substances (serotonin, adenosine diphosphate (ADP)), lipid derivatives (platelet aggregating factor (PAF), thromboxane $A_{2}$ $\left(\mathrm{TXA}_{2}\right)$ ), and other protein substances (collagen or immune complexes). The responses of platelets to agonists, known as platelet activation, include mainly adhesion (to foreign surfaces such as collagen or glass), shape change, aggregation and secretion of active compounds from three different types of storage granules (dense granules, $\alpha$-granules and lysosomes), shedding of microvesicles, development of platelet procoagulant activity and retraction of fibrin clots (Wu, 1996; Levy-toledano, 1999; Ryningen \& Holmsen, 1999). Blood platelets are involved in the hemostatic process. In hemostasis, platelet plug formation represents the primary response to vascular injury, with the coagulation cascade and fibrin formation. Normal primary hemostasis requires three critical events: platelet adhesion, secretion and aggregation. If a blood vessel is injured, platelets are exposed to subendothelial collagen. This initial interaction is mediated through the collagen receptor - integrin $\alpha_{2} \beta_{1}$, and by another platelet receptor - the glycoprotein complex GPIb/IX/V. Adherent platelets then change their shape from discs to spheres with pseudopods and release their granule contents (degranulation process). Few seconds later, more platelets are deposited on the collagen fibrils, and they start to stick to each other (platelet aggregation) (Wu, 1996; Levy-toledano, 1999; Ryningen \& Holmsen, 1999). Increased platelet activation with hyperaggregability is one of the risk factors in the pathogenesis of cardiovascular diseases.

Recently, it has been shown that homocysteine may modulate blood platelet functions (Rajkumar et al., 1999; Alexandru et al., 2007; Signorello et al., 2007; Olas et al., 2008; 2009). Some studies demonstrated that homocysteine promotes arachidonic acid release, the formation of thromboxane $\mathrm{A}_{2}$ (Signorello et al., 2002) and protein tyrosine phosphorylation in blood platelets (Leoncini et al., 2006; 2007). Results of Undas et al. (2007) demonstrated that the elevated total Hcy level is associated with increased platelet activation at the site of microvascular injury. McDonald et al. (1964) observed increased platelet adhesion in homocysteinuric patients. In an animal model of hyperhomocysteinemia (induced by a diet poor in folic acid), the aggregation of platelets stimulated by ADP or thrombin was higher than in control animals (Durand et al., 1996). Blood platelets obtained from patients with peripheral occlusive arterial disease and hyperhomocysteinemia, are more reactive and sensitive to agonists, but also far less sensitive to inhibitors (Riba et al., 2004). In diabetic patients, a high level of plasma Hcy is associated with more potent aggregation of blood platelets (Rajkumar et al., 1999). However, a direct action of Hcy and its derivatives on blood platelets is rather controversial. Some studies have reported that homocysteine alone does not induce platelet aggregation, but increases platelet aggregation induced by a strong platelet agonist — thrombin (Olas et al., 2008). Furthermore, these results suggest that this increase may be partly dependent on integrin $\alpha_{\text {II }} \beta_{3}$ activation on the platelet surface (Olas et al., 2008). It was also observed that the most reactive form of Hcy - HTL did not induce platelet aggregation on its own, but like Hcy, increased platelet aggregation induced by thrombin (Olas et al., 2008). In these studies authors used washed blood platelets (Olas et al., 2008). Other results showed that not only the reduced form of Hcy, but also HTL, may augment blood platelet activation; induced by other physiological agonists (ADP and collagen) and measured by flow cytometry or turbidimetry (Olas et al., 2009). These results (Olas et al., 2008; 2009) suggest that changes in the state of thiol groups in the platelet proteins, induced by Hcy or HTL, may potentiate aggregation stimulated by ADP or collagen. The tested concentrations of Hcy $(10-$ $100 \mu \mathrm{M})$ and HTL $(0.1-1 \mu \mathrm{M})$ used in studies of Olas et al. (2008 and 2009) corresponded to the levels found in plasma under hyperhomocysteinemia. Essex and Li (2003) observed the same process; when the effects of different low-molecular-weight thiols on platelet aggregation were tested. They found that not only glutathione at concentrations normally found in the blood, but also cysteine, cysteinylglycine and homocysteine potentiated platelet aggregation.

Recently, it has been suggested that N-homocysteinylated or S-homocysteinylated hemostatic proteins induced by Hcy or its thiolactone seem to be the main cause of the biotoxicity of homocysteine in cardiovascular diseases. The possibility that Hcy or HTL reacts with carbonyl groups present in human blood platelet proteins (Olas et al., 2009) and in human plasma proteins (Olas et al., 2010), also exists. In this context, the reaction of Hcy and HTL with carbonyl groups, for which the term C-homocysteinylation has been proposed, gains new significance. C-homocysteinylation induced by homocysteine or its thiolactone, may block protein degradation;; it may be also one of the pathomechanisms of some age-related diseases associated with hyperhomocysteinemia, such as Alzheimer disease, Parkinson disease and atherosclerosis.

Thiol homeostasis determines critical aspects of cell functions. Oxidation of thiol groups and reduction of the disulphide bonds in proteins is a dynamic, reversible process that occurs under physiological conditions in cells (Inayama et al., 2002; Martin et al., 2001). The concentration of thiol groups in proteins is much greater than that of glutathione; protein thiols are present as free thiols, disulphides, and mixed disulphides when conjugated with glutathione, cysteine, and $\gamma$-glutamylcysteine (Inayama et al., 2002). There is evidence that the metabolism of platelet protein thiols changed after incubation of platelets with Hcy and its thiolactone (Olas et al., 2009). It is suggested that Hcy may act as a platelet agonist, 
because it may act as an antagonist at the glycine site of the NMDA receptor (in the presence of normal or low glycine levels), or as an agonist at the glutamate site of this receptor (when glycine levels are increased) (Franconi et al., 1998; Morrell et al., 2008). Hcy, like other platelet agonists probably causes changes in protein disulphide isomerase (PDI) activity in platelets. Upon activation, platelets release PDI into the medium. This enzyme has been shown to be on the external surface of the platelet plasma membrane (Essex \& Li, 2003; Essex, 2004); it catalyses the formation as well as isomerisation of disulphide bonds. Essex and Li (2003) showed that PDI mediates platelet aggregation and secretion, and activates integrin $\alpha_{\mathrm{II}} \beta_{3}$; these findings suggest that this receptor is a target of PDI. PDI also denitrosates S-nitrosothiols, releasing nitric oxide $\left({ }^{\circ} \mathrm{NO}\right)$ that, via the guanylate cyclase/G-kinase route, attenuates platelet activation. Moreover, S-nitrosothiols are denitrosated at the same PDI-active site that catalyses the disulphide bond formation between integrins and their ligands, thereby attenuating irreversible aggregation.

The decrease in free $-\mathrm{NH}_{2}$ protein groups after 2-30 minutes of incubation of platelets with Hcy or HTL is a very important result of studies on blood platelets (Olas et al., 2009). At all the remaining times of incubation an increase in free amino groups was noted; this finding was inconsistent with the expected effect of N-homocysteinylation, which should decrease the number of free $\varepsilon$-amino groups. This may suggest that changes in the secondary protein structure induced by both Hcy and HTL are responsible for the exposure of new amino groups.

Blood platelets, in analogy to other circulating blood cells, generate reactive oxygen/nitrogen species (ROS/RNS) that may act as second messengers and regulate platelet functions. Accumulating evidence suggests a role of ROS/ RNS in platelet activation. On the other hand, an increased production of ROS/RNS causes oxidative stress, and thus, contributes to the development of various diseases, including vascular complications, inflammation, cancer and psychiatric illnesses (Olas \& Wachowicz, 2007). The results of our studies suggest that the reduced formation of nitric oxide ( $\mathrm{NO})$ in blood platelets treated with Hcy in a reduced form, or with HTL, may lead to an increase in platelet aggregation, because $\mathrm{NO}$ is a powerful aggregation inhibitor (Olas et al., 2008). It is known that homocysteine inhibits the synthesis of ${ }^{\circ} \mathrm{NO}$ in platelets and reduces ' $\mathrm{NO}$ bioavailability (Upchurch et al., 1997; Mutus et al., 2001; Undas et al., 2007); these effects of Hcy contribute to platelet hyperactivity and reduce tyrosine nitration in platelet proteins (Olas et al. 2008). The changes in protein tyrosine nitration may play an important role, since they may modulate tyrosine phosphorylation - a very important process for signal transduction in platelets. According to the results obtained by Olas et al. (2008), homocysteine and its thiolactone may influence the formation of the superoxide anion $\left(\mathrm{O}_{2}^{--}\right)$in blood platelets, as was estimated by the method of cytochrome c reduction. The described study showed that not only Hcy, but also HTL induces increased production of $\mathrm{O}_{2}^{-}{ }^{-}$both in resting and thrombin-activated platelets. Moreover, other results obtained by these authors (Olas et al., 2010) indicate that Hcy and its thiolactone may promote in vitro apoptotic events in human platelets.

\section{THE EFFECT OF HOMOCYSTEINE AND ITS THIOLACTONE ON ENDOTHELIAL CELLS}

At the cellular level the pathological role of homocysteine seems to be associated with alterations in en- dothelial cells, which play an important role in hemostasis. Endothelial cells are very sensitive even to a mild increase in Hcy concentration. This susceptibility may be explained by the fact; that human endothelial cells do not express the active form of cystathionine $\beta$-synthase, and consequently are not able to initiate homocysteine catabolism by the transsulfuration pathway (Jacobsen, 1998). The elevated level of Hcy may modulate functions of the vascular endothelium, changing the character of its surface from anticoagulant to procoagulant (Jacobsen, 1998). The anticoagulative properties of vascular endothelium are based on heparin-like glycosaminoglycanantithrombin III interactions. Results of Rodgers and Kane (1986) demonstrated a significant increase in the coagulation factor $\mathrm{V}$ activity, after exposure of endothelium to Hcy. Coagulation factor V in Hcy-modified endothelium is cleaved into fragments different than those obtained after factor $\mathrm{V}$ cleavage by thrombin or coagulation factor Xa (Rodgers \& Kane, 1986). Moreover, in hyperhomocysteinemia the prothrombotic tendency may be related to impaired inactivation of S-homocysteinylated coagulation factor $\mathrm{Va}$ by the activated protein $\mathrm{C}$ (Undas et al., 2001). It has been demonstrated that not only Hcy, but also HTL may modulate the properties and functions of endothelial cells. Results of Raposo et al. (2004) indicate that both compounds, Hcy and its thiolactone, inhibit the activity of lysyl oxidase (an enzyme involved in extracellular matrix maturation) in vascular endothelial cells. The report of Jakubowski et al. (2000) showed that protein N-homocysteinylation occurred in endothelial cells and that it depended on the concentration of Hcy. Modification of endothelial cell proteins may cause different pathophysiological consequences, such as modulation of the hemostasis system, that may contribute to the development of cardiovascular diseases. Detailed description of the effect of Hcy on endothelial cells has been provided by Karolczak and Olas (2009).

\section{HOMOCYSTEINE - FIBRONECTIN INTERACTION AND ITS CONSEQUENCES}

Human fibronectin (FN) is a multifunctional glycoprotein encoded by the FN gene on chromosome $2 \mathrm{q} 34$. This glycoprotein plays key roles in cell adhesion and migration, embryogenesis, differentiation, hemostasis, thrombosis, wound healing, as well as in tissue remodeling (Romberger, 1997). The FN subunit contains two fibrin binding sites. The site close to the amino terminus serves as a transglutamination site for activated factor XIII which crosslinks FN to various other proteins including fibrin, fibrinogen, and FN. The interaction with fibrin starts at the $\mathrm{N}$-terminal site of $\mathrm{FN}$ due to its higher affinity, followed by binding to the C-terminal site, which strengthens the fibrin-FN interaction. Interaction of FN with fibrin, mediated by factor XIII transglutaminase, is thought to be important for cell adhesion or cell migration into fibrin clots. After tissue injury, a blood clot formation serves the dual role of restoring vascular integrity and serving as a temporary scaffold for the wound healing process. Fibrin and plasma FN, the major protein components of blood clots, are essential to perform these functions. In the blood clotting process, after fibrin deposition, plasma FN-fibrin matrix is covalently crosslinked, and it then promotes fibroblast adhesion, spreading, and migration into the clot (Lee et al., 2010).

Fibronectin has free cysteine residues and numerous disulphide bonds that could interact with homocysteine via oxidative and/or thiol/disulphide exchange reactions. 
Majors et al. (2002) showed that homocysteine binds to several human plasma proteins, including fibronectin. The authors hypothesized that homocysteine binds to fibronectin via a disulphide linkage and that this binding results in a functional change, namely, the inhibition of fibrin binding by fibronectin. This inhibition may lead to a prolonged recovery from a thrombotic event and contribute to vascular occlusion.

\section{THE ROLE OF ANTIOXIDANTS IN HYPERHOMOCYSTEINEMIA}

Plasma homocysteine level depends among other things on sex, age, smoking, function of the liver and kidneys, physical activity and diet. Proper diet, abstaining from tobacco smoking, and optimal physical activity may influence the level of this amino acid. There are also various compounds which lead to the reduction of Hcy levels. Folic acid, vitamins $\mathrm{B}_{6}$ and $\mathrm{B}_{12}$ should be mentioned, because some pathways of Hcy metabolism are correlated with the level of these vitamins. Results of Pietrzik and Bronstrup (1998) showed that daily supplementation with folic acid in the range of $0.5-5 \mathrm{mg}$, and with about $0.5 \mathrm{mg}$ of vitamin $\mathrm{B}_{12}$ decreased Hcy concentration in blood. However, the results of Bogers et al. (2007) showed that increased fruit and vegetable consumption may be insufficient to change plasma Hcy concentration. Other experiments indicate that elevated homocysteine and folate deficiency are associated with oxidative stress. Oxidant injury has been suggested as a potential mechanism of atherogenesis in hyperhomocysteinemia (Sauls et al., 2007). Results of Kolling et al. (2011) demonstrated that supplementation of folic acid can be used as an adjuvant therapy in cardiovascular alterations caused by Hcy. They observed that Hcy induced oxidative-nitrative stress in a rat heart while folic acid had protective properties.

Epidemiologic studies indicate that regular consumption of polyphenolic antioxidants, the secondary plant metabolites, is correlated with a decreased risk of cardiovascular diseases, diabetes, arthritis and cancer. Moreover, on the basis of various observations, it is proposed that Hcy and HTL may act as oxidants in the model system in vitro and in vivo (Carluccio et al., 2007; Olas et al., 2008), but some dietary polyphenolic antioxidants can attenuate the oxidative damage- induced by hyperhomocysteinemia (Carluccio et al., 2007). A variety of well-known antioxidants, including polyphenolic antioxidants, have been shown to exert a protective action against Hcy toxicity (Schoecksnadel et al., 2005; Carluccio et al., 2007; Noll et al., 2009a; 2009b). However, the mechanisms of protection provided by many exogenous compounds against Hcy action are still unknown. Red wine-derived polyphenolic compounds at low doses significantly reduced plasma Hcy levels and restored the hepatic and plasma paraoxonase- 1 activity decreased in chronic hyperhomocysteinemia (Noll et al., 2009a; 2009b). Moreover, Noll et al. (2009a) observed that the aortic expression of proinflammatory cytokines and adhesion molecules as well as the level of the soluble lectin-like oxidized low-density lipoprotein receptor-1 were reduced in hyperhomocysteinemic mice fed with the red wine polyphenolic extract. Fu et al. (2003) reported that red wine prevents homocysteine - induced endothelial dysfunction in porcine coronary arteries. Our results showed that the well-studied polyphenol - resveratrol (3,4',5-trihydroxystilben), which is an integral component of human diet found naturally in fruits, nuts, flowers, seeds, red wine and bark of different plants, strongly, but not completely, reduced platelet apoptosis induced by Hcy or HTL, suggesting that pathways other than reactive oxygen species generation were also involved (Olas et al., 2010). Resveratrol exhibits a wide range of biological effects, including antiplatelet, anti-inflammatory, anticancer, antimutagenic and antifungal properties. It is also a potent antioxidant, reactive oxygen species scavenger and metal chelator. Resveratrol reduces lipid peroxidation; as well as oxidation and nitration of platelet and plasma proteins (Olas \& Wachowicz, 2005). Resveratrol and other polyphenolic compounds may also increase nitric oxide bioavailability, thereby antagonizing the development of endothelial dysfunction, decrease blood viscosity, improve insulin sensitivity, counteract platelet hyperactivity, inhibit platelet adhesion to fibrinogen-coated surfaces, as well as decrease plasma levels of von Willebrand factor, fibrinogen, and coagulation factor VII (Lippi et al., 2010). Furthermore, resveratrol may protect plasma proteins against modifications (measured by the level of thiol and $\varepsilon$-amino groups) caused by homocysteine or its thiolactone (Malinowska \& Olas, 2010). Resveratrol is also able to reduce the toxic action of Hcy and HTL on the hemostatic properties of fibrinogen and human plasma (Malinowska \& Olas, 2010). Since Hcy and its derivatives promote free radical production and oxidative damage to proteins (Sibrian-Vazques et al., 2010), it has been suggested that resveratrol, which can change the level of reactive oxygen species or reactive nitrogen species (specially that of nitric oxide), may be responsible for the reduction of protein modifications induced by Hcys or HTL. Results obtained by Malinowska and Olas (2011) also suggest that, due to such properties, resveratrol, may be responsible for the inhibition of platelet aggregation (stimulated by thrombin) during hyperhomocysteinemia.

Grape seeds are one of the richest plant sources of phenolic substances, and Kolodziejczyk et al. (2011) observed that the grape seed extract reduced the toxic effect of Hcys and HTL on fibrinolysis. The grape seed extract $(12.5-50 \mu \mathrm{g} / \mathrm{ml})$ supported plasminogen to plasmin conversion inhibited by Hcys or HTL. For example, in the presence of grape seed extract (at the highest tested concentration $-50 \mu \mathrm{g} / \mathrm{ml}$ ) the increase of about $78 \%$ (for human plasminogen-treated with Hcys) and $56 \%$ (for human plasma-treated with Hcys), was found. These studies also demonstrated in the in vitro model system, that the grape seed extract (12.5-50 $\mu \mathrm{g} /$ $\mathrm{ml}$ ) diminished the reduction of thiol groups and of lysine $\varepsilon$-amino groups in plasma proteins (as determined via colorimetric methods) treated with Hcys $(0.1 \mathrm{mM})$ or HTL $(1 \mu \mathrm{M})$. In the presence of the grape seed extract at the concentration of $50 \mu \mathrm{g} / \mathrm{ml}$, the level of reduction of thiol groups reached about $45 \%$ (for plasma treated with Hcys) and about 15\% (for plasma treated with HTL).Very similar protective effects of the grape seed extract were observed in the measurements of lysine $\varepsilon$-amino groups in plasma proteins treated with Hcys or HTL (Kołodziejczyk et al., 2011). Our preliminary results indicate that the extract from berries of Aronia melanocarpa (a rich source of phenolic substances) reduces the toxic effects of Hcy and HTL on the hemostatic properties of fibrinogen and plasma. These findings indicate a possible protective action of the $A$. melanocarpa extract in hyperhomocysteinemia-induced cardiovascular disorders. Moreover, our experiments showed that the extract from berries of $A$. melanocarpa, due to its antioxidant action, significantly attenuated 


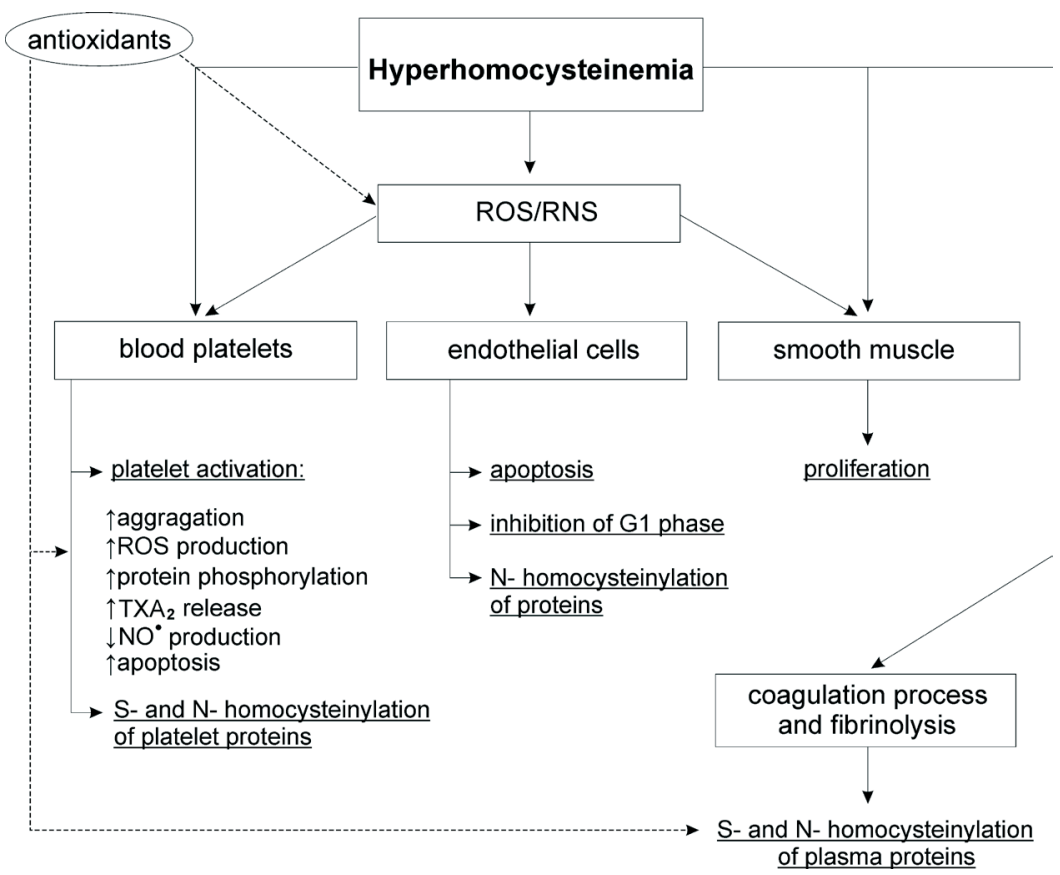

Figure 5. Proposed model for the protective role of phenolic antioxidants on selected elements of hemostasis during hyperhomocysteinemia.

Abbreviations: RNS, reactive nitric species; ROS, reactive oxygen species; $\cdot N O$, nitric oxide, $T_{X A_{2}}$ - thromboxane $A_{2}$. the oxidative stress (assessed by measuring of the total antioxidant status — TAS) in plasma in a model of hyperhomocysteinemia (Malinowska et al., 2011). In comparative studies, the extract from berries of $A$. melanocarpa and resveratrol had similar protective properties (Malinowska et al., 2011). Other available data suggest that high dietary intake of antioxidative vitamins (vitamin A, beta-carotene and vitamin C) could be a protective factor against atherosclerosis and the pro-oxidative effect of Hcy on low density lipoproteins (LDL) (Seo et al., 2010). It has been established that Hcy alters glutamate uptake, $\mathrm{Na}^{+} / \mathrm{K}^{+}$-ATPase activity and the oxidative status in rat hippocampus, while vitamin $\mathrm{C}$ prevents these effects (Machado et al., 2011). More information about the role of diet in prophylaxis and treatment of hyperhomocysteinemia has been provided by Malinowska et al. (2010) and Manolescu et al. (2010).

\section{CONCLUSIONS}

The contribution of hyperhomocysteinemia to hemostatic abnormalities is complex and still unclear. Hcy or its derivatives, e.g. its thiolactone, may modulate the signal transduction in different types of blood cells and sometimes act in opposite ways. Homocysteine and HTL also cause changes in the level of the reactive oxygen and nitrogen species (particularly -NO) and may be responsible for the modification of hemostasis induced by these compounds. Moreover, the biological significance of hemostatic protein modifications (fibrinogen and other coagulation factors) induced by Hcy or HTL is only partly recognized. It has been established, however, that in particular, Nhomocysteinylation induced by HTL may play an important role in different pathophysiological anomalies leading to cardiovascular diseases. This review article describes the current knowledge on the prevention of the negative consequences of hyperhomocysteinemia. It seems that various antioxidants (present in human diet), including phenolic compounds, may reduce the toxic effects of Hcy or its derivatives on hemostasis (Fig. 5). These findings give hope for the develop- ment of dietary supplements, which will be capable of preventing thrombosiss which occurs under pathological conditions, observed also in hyperhomocysteinemia, such as plasma procoagulant activity and oxidative stress.

\section{Acknowledgement}

This work was supported by grant (No 0365/B/ PO1/2011/40) from the National Science Centre (Poland).

\section{Conflicts of interest disclosure}

The Authors state that they had no interests which might be perceived as posing a conflict or bias.

\section{REFERENCES}

Acevedo M, Pearce GL, Kottke-Marchant K, Sprecher DL (2002) Elevated fibrinogen and homocysteine levels enhance the risk of mortality in patients from a high-risk preventive cardiology clinic. Arterioscler Thromb Vasc Biol 22: 1042-1045.

Alexandru N, Jardin I, Popov D, Simionescu M, Garcia-Estan J, Salido GM, Rosado JA (2007) Effect of homocysteine on calcium mobilization and platelet function in type 2 diabetes mellitus. J Cell Mol Med 14: 111-117.

Bednarek-Tupikowska G, Tupikowski K (2004) Homocysteine- an underestimated atheromatosis risk factor. Do sex hormones influence homocysteine concentrations. Postepy Hig Med Dosw 58: 381-389.

Bełtowski J (2005) Protein homocysteinylation: a new mechanism of atherogenesis? Postęy Higieny Med Dośw 59: 394-404 (in Polish).

Blom HJ, Smulders Y (2011) Overview of homocysteine and folate metabolism. With special references to cardiovascular disease and neural tube defects. J Inherit Metab Dis 34: 75-81.

Bogers RP, Dagnelie PC, Bast A, van Leeuwen M, van Klaveren JD, van den Brandt PA (2007) Effect of increased vegetable and fruit consumption on plasma folate and homocysteine concentrations. Nutrition 23: 97-102.

Booth NA (2000) Regulation of fibrinolytic activity by localization of inhibitors to fibrin(ogen). Fibrinolysis \& Proteolysis 14: 206-213.

Boysen G, Brander T, Christensen H, Gideon R, Truelsen T (2003) Homocysteine and risk of recurrent stroke. Stroke 34: 1258-1261.

Brattstörm L, Wilcken DE, Ohrvik J, Brudin L (1998) Common methylenetetrahydrofolate reductase gene mutation leads to hyperhomocysteinemia but not to vascular disease: The result of a metaanalysis. Circulation 98: 2520-2526. 
Cacciapuoti F (2011) Hyper-homocysteinemia: a novel risk factor or a powerful marker for cardiovascular diseases? Pathogenetic and therapeutical uncertainties. J Thromb Thrombolysis 32: 82-88.

Carluccio MA, Ancora MA, Massaro M, Carluccio M, Scoditti E, Distante A, Storelli C, De Caterina R (2007) Homocysteine induces VCAM-1 gene expression through NF-kappaB and NAD(P)H oxidase activation: protective role of Mediterranean diet polyphenolic antioxidants. Am I Physiol Heart Circ Physiol 293: 2344-2354.

Chango A, Boisson F, Barbe F, Quilliot D, Droesch S, Pfister M, Fillon-Emery N, Lambert D, Frémont S, Rosenblatt DS, Nicolas JP (2000) The effect of $677 \mathrm{C} \rightarrow \mathrm{T}$ and $1298 \mathrm{~A} \rightarrow \mathrm{C}$ mutations on plasma homocysteine and 5,10-methylenetetrahydrofolate reductase activity in healthy subjects. BrJ Nutr 83: 593-596.

Chwatko G, Jakubowski H (2005a) The determination of homocysteine-thiolactone in human plasma. Anal Biochem 337: 271-277.

Chwatko G, Jakubowski H (2005b) Urinary extraction of homocysteine thiolactone in humans. Clin Chem 51: 408-415.

Colucci M, Cattaneo M, Martinelli I, Semeraro F, Binetti BM, Semeraro N (2008) Mild hyperhomocysteinemia is associated with increased TAFI levels and reduced plasma fibrinolytic potential. Journal of Thrombosis and Haemostasis 6: 1571-1577.

D’Angelo A, Selhub J (1997) Homocysteine and thrombotic disease. Blood 90: 1-11.

Dassah M, Deora AB, He K, Hajjar KA (2009) The endothelial cell annexin A2 system and vascular fibrinolysis. Gen Physiol Biophys 28: 20-28.

Di Minno MND, Tremoli E, Coppola, Lupoli R, Di Minno G (2010) Homocysteine and arterial thrombosis: Challenge and opportunity. Thromb Haemost, 103: 942-961.

Durand P, Prost M, Blache D (1996) Pro-thrombotic effects of a folic acid deficient diet in rat platelets and macrophages related to elevated homocysteine and decreased n-3 polyunsaturated fatty acids. Atherosclerosis 121: 231-243.

Essex DW (2004) Platelet surface glutathione reductase-like activity. Blood 104: 12383-12385.

Essex DW, Li M (2003) Redox control of platelet aggregation. Biochemistry 42: 129-136.

Franconi F, Miceli M, Alberti L, Seghieri G, De Montis MG, Tagliamonte A (1998) Further insights into the anti-aggregating activity of NMDA in human platelets. Br J Pharmacol 124: 35-40.

Franken DG, Boers GHJ, Blom HJ, and Trijbels JMF (1994) Effect of various regimens of vitamin $\mathrm{B}_{6}$ and folic acid on mild hyperhomocysteinaemia in vascular patients. I Inherited Metab Dis 17: 159-162.

Freyburger G, Labrouche S, Sassoust G, Rouanet K, Javorschi S, Parrot F (1997) Mild hyperhomocysteinemia and hemostatic factors in patients with arterial vascular diseases. Thromb Haemost 77: 466-471.

Fu W, Conklin BS, Lin PH, Lumsden AB, Yao Q, Chen C (2003) Red wine prevents homocysteine-induced endothelial dysfunction in porcine coronary arteries. I Surg Res 115: 82-91.

Glowacki R, Jakubowski H (2004) Cross-talk between Cys34 and lysine residues in human serum albumin revealed by N-homocysteinylation. I Biol Chem 279: 10864-10871.

Hajjar KA, Mauri L, Jacovina AT, Zhong F, Mirzai UA, Padovani JC, Chait BT (1998) Tissue plasminogen activator binding to the annexin II tail domain. J Biol Chem 273: 9987-9993.

Harpel PC, Chang VT, Borth W (1992) Homocysteine and other sulfhydryl compounds enhance the binding of lipoprotein(a) to fibrin: a potential biochemical link between thrombosis, atherogenesis, and sulfhydryl compound metabolism. Proc Natl Acad Sci USA 89: 10193-10197.

Harpel PC, Zhang X, Borth W (1996) Homocysteine and haemostasis: pathogenic mechanisms predisposing to thrombosis. I Nutr 126: 1285S-1289S

Hop CE, Bakhtiar R (2002) Homocysteine thiolactone and protein homocysteinylation: mechanistic studies with model peptides and proteins. Rapid Commun Mass Spectrom 16: 1049-1053.

Inayama T, Oka J, Kashiba M, Saito M, Higuchi M, Umegaki K, Yamamoto Y, Matsuda M (2002) Moderate physical exercise induces the oxidation of human blood protein thiols. Life Sci 70: 2039-2046.

Jacobsen DW (1998) Homocysteine and vitamins in cardiovascular disease. Clin Chem 44: 1833-1843.

Jakubowski H (1990) Proofreading in vivo: editing of homocysteine by methionyl-tRNA synthetase in Escherichia coli. Proc Natl Acad Sci USA 87: 4504-4508.

Jakubowski H (1999) Protein homocysteinylation: possible mechanism underlying pathological consequences of elevated homocysteine levels. Faseb J 13: 2277-2283.

Jakubowski H (2000) Calcium-dependent human serum homocysteine thiolactone hydrolase. A protective mechanism against protein $\mathrm{N}$ homocysteinylation. I Biol Chem 275: 3957-3362.

Jakubowski H (2002) Homocysteine is a protein amino acid in humans. Implications for homocysteine-linked disease. J Biol Chem 277: 30425-30428.

Jakubowski H (2003) Homocysteine-thiolactone and S-nitroso-homocysteine mediate incorporation of homocysteine into protein in humans. Clin Chem Lab Med 41: 1462-1466.
Jakubowski H (2004) Molecular basis of homocysteine toxicity in humans. Cell Mol Life 61: 470-487.

Jakubowski H (2005) Anti-N-homocysteinylated protein autoantibodies and cardiovascular disease. Clin Chem Lab Med 43: 1011-1014.

Jakubowski H (2006) Pathophysiological consequences of homocysteine excess. J Nutr 136: 1741S-1749S.

Jakubowski H (2010) The role of paraoxonase 1 in the detoxification of homocysteine thiolactone. Adv Exp Med Biol 660: 113-127.

Jakubowski H, Głowacki R (2011) Chemical biology of homocysteine thiolactone and related metabolites. Adv Clin Chem 55: 81-103.

Jakubowski H, Zhang L, Bardeguez A, Aviv A (2000) Homocysteine thiolactone and protein homocysteinylation in human endothelial cells: implications for atherosclerosis. Circ Res 87: 45-51.

Kamat GV, Metgud SC, Pattanshetti VM, Godhi AS (2010) A crosssectional study to detect the prevalence of hyperhomocysteinemia in cases of deep vein thrombosis. Indian J Surg 72(4): 323-326.

Kang SS, Wong PW, Malinow MR (1992) Hyperhomocyst(e)inemia as a risk factor for occlusive vascular disease. Annu Rev Nutr 12: 279-289.

Karolczak K, Olas B (2009) Mechanism action of homocysteine and its thiolactone in haemostasis system. Physiol Res 58: 623-633.

Klerk M, Verhoef P, Clarke R, Blom HJ, Kok FJ, Schouten EG, MTHFR Studies Collaboration Group (2002) MTHFR $677 \mathrm{C} \rightarrow \mathrm{T}$ polymorphism and risk of coronary heart disease: meta-analysis. JAMA 288: 2023-2031.

Kolling J, Scherer EB, da Cunha AA, da Cunha MJ, Wyse AT (2011) Homocysteine induces oxidative-nitrative stress in heart of rats: prevention by folic acid. Cardiovasc Toxicol 11: 67-73.

Kolodziejczyk J, Malinowska J, Nowak P, Olas B (2010) Comparison of the effect of homocysteine and its thiolactone on the fibrinolytic system using human plasma and purified plasminogen. Mol Cell Biochem 344: 217-220.

Kolodziejczyk J, Malinowska J, Olas B, Stochmal A, Oleszek W, Erler J (2011) The polyphenol-rich extract from grape seeds suppresses toxicity of homocysteine and its thiolactone on the fibrinolytic system. Thromb Res 127: 489-491.

Lee J, Ku Y, Rhyu I, Chung Ch, Park Y (2010) Effects of fibrin-binding oligopeptide on osteopromotion in rabbit calvarial defects. I Periodontal Implant Sci 40: 211-219.

Lentz SR, Erger RA, Dayal S, Maeda N, Malinow MR, Heistad DD, Faraci FM (2000) Folate dependence of hyperhomocysteinemia and vascular dysfunction in cystathionine beta-synthase-deficient mice. Am I Physiol 279: 970-975.

Lentz SR, Haynes WG (2004) Homocysteine: It is a clinically important cardiovascular risk factor? Clev Clin I Med 71: 729-734.

Leoncini G, Bruzzese D, Signorello MG (2006) Activation of p38MAPKinase/cPLA 2 pathway in homocysteine treated platelets. I Thromb Haemos 4: 209-216.

Leoncini G, Bruzzese D, Signorello MG (2007) A role for PLC $\gamma 2$ in platelet activation by homocysteine. J Cell Biochem 100: 1255-1265.

Levy-Toledano S (1999) Platelet signal transduction pathways: Could we organize them into a 'hierarchy'? Haemostasis 29: 4-15.

Li Z, Sun L, Zhang H, Liao Y, Wang D, Zhao B, Zhu Z, Zhao J, Ma A, Han Y (2003) Elevated plasma homocysteine was associated with hemorrhagic and ischemic stroke, but methylenetetrahydrofolate reductase gene C677T polymorphism was a risk factor for thrombotic stroke: a Multicenter Case-Control Study in China. Stroke 34: 2085-2090.

Lijnen HR (2002) Non-haemostatic role of blood coagulation proteases and their receptors. Biochemical Society Transactions 30: 1631-1667.

Lippi G, Franchini M, Favaloro EJ, Targher G (2010) Moderate red wine consumption and cardiovascular disease risk: beyond the "French paradox". Semin Thromb Hemost 36: 59-70.

Machado FR, Ferreira AG, da Cuncha AA, Tagliari B, Mussulini BH, Wofchuk S, Wyse AT (2011) Homocysteine alters glutamate uptake and $\mathrm{Na}^{+}, \mathrm{K}^{+}-\mathrm{ATPase}$ activity and oxidative status in rats hippocampus: protection by vitamin C. Metab Brain Dis 26: 61-67.

Majors AK, Sengupta S, Willard B, Kinter MT, Pyeritz RD, Jacobsen DW (2002) Homocysteine binds to human plasma fibronectin and inhibits its interaction with fibrin. Arterioscler Thromb Vasc Biol 22: 1354-1359.

Malinowska J, Babicz K, Olas B, Stochmal A, Oleszek W (2011) Aronia melanocarpa extract suppresses biotoxicity of homocysteine and its metabolite on hemostatic activity of fibrinogen and plasma. $\mathrm{Nu}$ trition 1-6 (in press).

Malinowska J, Kolodziejczyk J, Olas B (2010) Prophylaxis and treatment of hyperhomocysteinemia. Progress in Medicine 23: 571-576.

Malinowska J, Nowak P, Olas B (2011) Comparison of the effect of homocysteine in the reduced form, its thiolactone and protein homocysteinylation on hemostatic properties of plasma. Thromb Res 127: 214-219.

Malinowska J, Olas B (2010) Effect of resveratrol on hemostatic properties of human fibrinogen and plasma during model of hyperhomocysteinemia. Thromb Res 126: 379-382.

Malinowska J, Olas B (2011) Response of blood platelets to resveratrol during model of hyperhomocysteinemia. Platelets 22: 277-283. 
Manolescu BN, Oprea E, Farcasanu IC, Berteanu M, Cercasov C (2010) Homocysteine and vitamin therapy in stroke prevention and treatment: a review. Acta Biochim Pol 57: 467-477.???? 4

Mansoor MA, Svardal AM, Ueland PM (1992) Determination of the in vivo redox status of cysteine, cysteinylglycine, homocysteine, and glutathione in human plasma. Anal Biochem 200: 218-229.

Marczak L, Sikora M, Stobiecki M, Jakubowski H (2011) Analysis of site-specific N-homocysteinylation of human serum albumin in vitro and in vivo using MALDI-ToF and LC-MS/MS mass spectrometry. J Proteomics 74: 967-974.

Martin C, Martinez R, Navarro R, Ruiz-Sanz JI, Lacort M, Ruiz-Larrea MB (2001) tert-Butyl hydroperoxide-induced lipid signalling in hepatocytes: involvement of glutathione and free radicals. Biochem Pharmacol 62: 705-712.

Mc Donald L, Bray C, Field C, Love F, Davies B (1964) Homocystinuria, thrombosis and the blood platelets. Lancet 1: 745-746.

Morrell CN, Sun H, Ikeda M, Beique JC, Swaim AM, Mason E, Martin TV, Thompson LE, Gozen O, Ampagoomian D, Sprengel R, Rothstein J, Faraday N, Huganir R, Lowenstein CJ (2008) Glutamate mediates platelet activation through the AMPA receptor. $J$ Exp Med 205: 575-584.

Mutus B, Rabini RA, Staffolani R, Ricciotti R, Fumelli P, Moretti N, Martarelli D, Mazzanti L (2001) Homocysteine-induced inhibition of nitric oxide production in platelets: A study on healthy and diabetic subjects. Diabetologia 44: 979-982.

Nardulli M, Durlach V, Pepe G, Anglés-Cano E (2005) Mechanism for the homocysteine-enhanced antifibrinolytic potential of lipoprotein(a) in human plasma. Thromb Haemost 94: 75-81.

Noll C, Hamelet J, Ducros V, Belin N, Paul JL, Delabar JM, Janel N (2009) Resveratrol supplementation worsen the dysregulation of genes involved in hepatic lipid homeostasis observed in hyperhomocysteinemic mice. Food Chem Toxicol 47: 230-236.

Noll C, Hamelet J, Matulewicz E, Paul JL, Delabar JM, Janel N (2009) Effects of red wine polyphenolic compounds on paraoxonase-1 and lectin-like oxidized low-density lipoprotein receptor-1 in hyperhomocysteinemic mice. I Nutr Bichem 20: 586-596.

Nygard O, Nordrehaug JE, Refsum H, Ueland PM, Farstad M, Vollset SE (1997) Plasma homocysteine levels and mortality in patients with coronary artery disease. N Engl J Med 337: 230-236.

Olas B, Kedzierska M, Wachowicz B (2008) Comparative studies on homocysteine and its metabolite - homocysteine thiolactone action in blood platelets in vitro. Platelets 19: 520-527.

Olas B, Kolodziejczyk J, Kedzierska M, Rywaniak J, Wachowicz B (2009) Modification of human blood platelet induced by homocysteine and its thiolactone in vitro. Thromb Res 124: 689-94.

Olas B, Kolodziejczyk J, Malinowska J (2010) May modifications of human plasma proteins stimulated by homocysteine and its thiolactone induce changes of haemostatic function of plasma in vitro? Gen Physiol Biophys 29: 186-193.

Olas B, Malinowska J, Rywaniak J (2010) Homocysteine and its thiolactone may promote apoptotic events in blood platelets in vitro. Platelets 21: 533-540.

Olas B, Wachowicz B (2005) Resveratrol, a phenolic antioxidant with effects on blood platelet functions. Platelets 16: 251-260.

Olas B, Wachowicz B (2007) Role of reactive nitrogen species in blood platelet functions. Platelets 23: 1-11.

Perla-Kaján J, Jakubowski H (2010) Paraoxonase 1 protects against protein N-homocysteinylation in humans. FASEB J 24: 931-936.

Perła-Kajan J, Twardowski T, Jakubowski H (2007) Mechanisms of homocysteine toxicity in humans. Amino Acids 32: 561-572.

Pietrzik K, Bronstrup A (1998) Vitamins B12, B 6 and folate as determinants of homocysteine concentration in the healthy population. Eur I Pediatr 157: 135-138.

Rajkumar V, Ragatzki P, Sima A, Levy J (1999) Enhanced platelet aggregation, high homocysteine level, and microvascular disease in diabetic muscle infarctions: Implications for therapy. Endocrine 11: 57-60.

Ramakrishnan S, Sulochana KN, Lakshmi S, Selvi R, Angayarkanni K (2006) Biochemistry of homocysteine in health and diseases. Indian J Biochem Biophys 43: 275-283.

Raposo B, Rodriguez C, Martinez-Gonzales J, Badimon L (2004) High levels of homocysteine inhibit lysyl oxidase (LOX) and downregulate LOX expression in vascular endothelial cells. Atheroscl 177: 1-8.

Ravaglia G, Forti P, Maioli F, Martelli M, Servadei L, Brunetti N, Porcellini E, Licastro F (2005) Homocysteine and folate as risk factors for dementia and Alzheimer disease. Am J Clin Nutr 82: 636-664.

Riba R, Nicolaou A, Troxler M, Homer-Vaniasinkam S, Naseem KM (2004) Altered platelet reactivity in peripheral vascular disease complicated with elevated plasma homocysteine levels. Atheroscl 175: 69-75.

Rodgers GM, Kane WH (1986) Activation of endogenous factor V by a homocysteine-induced vascular endothelial cell activator. J Clin Invest 77: 1909-1916.
Romberger DJ (1997) Fibronectin. Int J Biochem Cell Biol 29: 939-943.

Ryningen A, Holmsen H (1999) In Platelet Physiology and Pharmacology. Gundu H, Rao R, red, pp 1-22. Kluwer Academic Publishers Norwell.

Sauls DL, Arnold EK, Bell CW, Allen JC, Hoffman M (2007) Prothrombotic and pro-oxidant effects of diet-induced hyperhomocysteinemia. Thromb Res 120: 117-126.

Sauls DL, Lockhart E, Warren ME, Lenkowski A, Wilhelm S, Hoffman M (2006) Modification of fibrinogen by homocysteine thiolactone increases resistance to fibrinolysis: a potential mechanism of the thrombotic tendency in hyperhomocysteinemia. Biochem 45: 2480-2487.

Sauls DL, Wolberg AS, Hoffman M (2003) Elevated plasma homocysteine leads to alterations in fibrin clot structure and stability: Implications for the mechanism of thrombosis in hyperhomocysteinemia. J Thromb Haemost 1: 300-306.

Sawula W, Banecka-Majkutewicz Z, Kadziński L, Jakobkiewicz-Banecka J, Wegrzyn G, Nyka W, Banecki B (2009) Homocysteine lev$\mathrm{el}$ and metabolism in ischemic stroke in the population of Northern Poland. Clinical Biochemistry 42: 442-447.

Schoecksnadel K, Winkler C, Wirleitner B, Schennach H, Weiss G, Fuchs D (2005) Anti-inflammatory compound resveratrol suppresses homocysteine formation in stimulated human peripheral blood mononuclear cells in vitro. Clin Chem Lab Med 43: 1084-1088.

Sengupta S, Chen H, Togawa T, Dibello PM, Majors K, Budy B, Ketterer ME, Jacobsen DW (2001) Albumin thiolate anion is an intermediate in the formation of albumin-S-S-homocysteine. J Biol Chem 276: 30111-30117.

Seo H, Oh H, Park H, Jang Y, Lee M (2010) Contribution of dietary of antioxidants to homocysteine-induced low density lipoprotein (LDL) oxidation in atherosclerotic patients. Yonsei Med J 51: 526533.

Sibrian-Vazquez M, Escobedo JO, Lim S, Samoei GK, Strongin RM (2010) Homocystamides promote free-radical and oxidative damage to proteins. Proc Natl Acad Sci USA 107: 551-554.

Signorello MG, Pascale R, Leoncini G (2002) Effect of homocysteine on arachidonic acid release in human platelets. Eur J Clin Invest 32: 279-284.

Signorello MG, Viviani GL, Armani U, Cerone R, Minniti G, Piana A, Leoncini $G$ (2007) Homocysteine, reactive oxygen species and nitric oxide in type 2 diabetes mellitus. Thromb Res 120: 607-613.

Sikora M, Marczak L, Twardowski T, Stobiecki M, Jakubowski H (2010) Direct monitoring of albumin lysine-525 N-homocysteinylation in human serum by liquid chromatography/mass spectrometry. Anal Biochem 405: 132-134.

Speidl WS, Nikfardjam M, Niessner A, Zeiner A, Jordanova N, Zorn G, Maurer G, Schreiber W, Wojta J, Huber K (2007) Mild hyperhomocysteinemia is associated with a decreased fibrinolytic activity in patients after ST-elevation myocardial infarction. Thromb Res 119: 331-336.

Suwala W, Banecka-Majkutewicz Z, Kadziński L, Jakóbkiewicz-Banecka J, Wegrzyn G, Nyka W, Banecki B (2008) Improved HPLC method for total plasma homocysteine detection and quantification. Acta Biochim Pol 55: 119-125.

Undas A, Brozek J, Jankowski M, Siudak Z, Szczeklik A, Jakubowski H (2006) Plasma homocysteine affects fibrin clot permeability and resistance to lysis in human subjects. Arterioscler Thromb Vasc Biol 26: 1397-1404.

Undas A, Stepien E, Plicner D, Zielinski L, Tracz W (2007) Elevated total homocysteine is associated with increased platelet activation at the site of microvascular injury. Effects of folic acid administration. J Thromb Haemost 5: 1070-1072.

Undas A, Williams EB, Butenas S, Orfeo T, Mann KG (2001) Homocysteine inhibits inactivation of factor $\mathrm{Va}$ by activated protein $\mathrm{C} . J$ Biol Chem 276: 4389-4397.

Upchurch GRJ, Welch GN, Fabian AJ, Freedman JE, Johnson JL, Keaney JFJ, Loscalzo J (1997) Homocyst(e)ine decrease biovailable nitric oxide by a mechanism involving glutathione peroxidase. $J$ Biol Chem 272: 17012-17017.

Wu K (1996) Platelet activation mechanisms and marker in arterial thrombosis. J Inter Med 239: 17-34.

Xue S, Green MA, LoGrasso PV, Boettcher BR, Madison EL, Curtiss LK, Miles LA (1999) Comparison of the effects of Apo(a) kringle IV-10 and plasminogen kringles on the interactions of lipoprotein(a) with regulatory molecules. Thromb Haemost 81: 428-435.

Zimny J (2008) Mechanisms that protect against homocysteine toxicity. Progress in Biochemistry 54: 91-96.

Zou CG, Banerjee R (2005) Homocysteine and redox signaling. Antioxid Redox Signal 7: 47-59. 